\title{
Histone deacetylases 1 and 3 but not 2 mediate cytokine-induced beta cell apoptosis in INS-1 cells and dispersed primary islets from rats and are differentially regulated in the islets of type 1 diabetic children
}

\author{
M. Lundh • D. P. Christensen • M. Damgaard Nielsen • \\ S. J. Richardson • M. S. Dahllöf • T. Skovgaard • \\ J. Berthelsen - C. A. Dinarello • A. Stevenazzi • \\ P. Mascagni • L. G. Grunnet • N. G. Morgan • \\ T. Mandrup-Poulsen
}

Received: 26 February 2012 / Accepted: 10 May 2012 / Published online: 7 July 2012

(C) Springer-Verlag 2012

\begin{abstract}
Aims/hypothesis Histone deacetylases (HDACs) are promising pharmacological targets in cancer and autoimmune diseases. All 11 classical HDACs (HDAC1-11) are found in the pancreatic beta cell, and HDAC inhibitors (HDACi) protect beta cells from inflammatory insults. We investigated which HDACs mediate inflammatory beta cell damage
\end{abstract}

D. P. Christensen and M. Damgaard Nielsen contributed equally to this study.

Electronic supplementary material The online version of this article (doi:10.1007/s00125-012-2615-0) contains peer-reviewed but unedited supplementary material, which is available to authorised users.

M. Lundh $(\bowtie) \cdot$ D. P. Christensen · M. Damgaard Nielsen • M. S. Dahllöf • L. G. Grunnet · T. Mandrup-Poulsen

Center for Medical Research Methodology, Department of

Biomedical Sciences, Faculty of Health and Medical Sciences,

University of Copenhagen,

Blegdamsvej 3,

2200 Copenhagen, Denmark

e-mail: morlun@sund.ku.dk

M. Damgaard Nielsen · T. Skovgaard · J. Berthelsen

The Novo Nordisk Foundation Center for Protein Research, Faculty of Health and Medical Sciences,

University of Copenhagen,

Copenhagen, Denmark

\section{S. J. Richardson}

Peninsula Medical School, University of Plymouth,

Plymouth, UK

\section{A. Dinarello}

Department of Medicine, University of Colorado Denver,

Aurora, CO, USA and how the islet content of these HDACs is regulated in recent-onset type 1 diabetes.

Methods The rat beta cell line INS-1 and dispersed primary islets from rats, either wild type or HDAC1-3 deficient, were exposed to cytokines and HDACi. Molecular mechanisms were investigated using real-time PCR, chromatin immunoprecipitation and ELISA assays.
L. G. Grunnet

Novo Nordisk,

Måløv, Denmark

N. G. Morgan

Peninsula Medical School, University of Exeter,

Plymouth, UK

\section{T. Mandrup-Poulsen}

Department of Molecular Medicine and Surgery,

Karolinska Institute,

Stockholm, Sweden 
Pancreases from healthy children and children with type 1 diabetes were assessed using immunohistochemistry and immunofluorescence.

Results Screening of 19 compounds with different HDAC selectivity revealed that inhibitors of HDAC1, 2 and -3 rescued INS- 1 cells from inflammatory damage. Small hairpin RNAs against HDAC1 and -3 , but not HDAC2, reduced pro-inflammatory cytokine-induced beta cell apoptosis in INS-1 and primary rat islets. The protective properties of specific HDAC knock-down correlated with attenuated cytokine-induced iNos expression but not with altered expression of the proinflammatory mediators $I l 1 \alpha, I l 1 \beta$, Tnf $\alpha$ or Cxcl2. HDAC3 knock-down reduced nuclear factor $\mathrm{kB}$ binding to the iNos promoter and HDAC1 knock-down restored insulin secretion. In pancreatic sections from children with type 1 diabetes of recent onset, HDAC1 was upregulated in beta cells whereas $\mathrm{HDAC} 2$ and -3 were downregulated in comparison with five paediatric controls.

Conclusions/interpretation These data demonstrate nonredundant functions of islet class I HDACs and suggest that targeting HDAC1 and HDAC3 would provide optimal protection of beta cell mass and function in clinical islet transplantation and recent-onset type 1 diabetic patients.

Keywords Autoimmunity $\cdot$ Cytokines $\cdot$ Epigenetics · HDAC · HDAC1 - HDAC2 - HDAC3 - Inflammation · $\mathrm{NF} \kappa \mathrm{B} \cdot$ Type 1 diabetes

$\begin{array}{ll}\text { Abbreviations } \\ \text { ChIP } & \text { Chromatin immunoprecipitation } \\ \text { ECM } & \text { Extracellular matrix } \\ \text { ER } & \text { Endoplasmic reticulum } \\ \text { HC } & \text { Helminthosporium carbonum } \\ \text { HDAC } & \text { Histone deacetylase } \\ \text { HDACi } & \text { Histone deacetylase inhibitors } \\ \text { MOI } & \text { Multiplicity of infection } \\ \text { NFKB } & \text { Nuclear factor } \mathrm{KB} \\ \text { shRNA } & \text { Small hairpin RNA }\end{array}$

\section{Introduction}

Beta cell function in type 1 diabetes of recent onset can be preserved by controlling the autoimmune process with the potent $\mathrm{T}$ cell immunosuppressant ciclosporin, but clinical use of this drug is prohibited by the need for long-term administration and the ensuing adverse effects [1]. Short courses of anti-CD3 or anti-CD20 antibodies with specific actions on $\mathrm{T}$ or B lymphocyte functions, respectively, preserve beta cell function in recent-onset type 1 diabetes, but the effects are transient $[2,3]$, and depletion of $\mathrm{T}$ and $\mathrm{B}$ cells increases the risk of developing progressive multifocal leucoencephalopathy [4]. There is therefore a need for safe, preferably orally administered, drugs that can effectively control islet inflammation.

Orally active and clinically well-tolerated broad inhibitors of histone deacetylases (HDACs), a class of enzymes that catalyse the deacetylation of cytosolic and nuclear proteins, including histones and transcription factors such as nuclear factor $\mathrm{\kappa B}(\mathrm{NF} \kappa \mathrm{B})[5,6]$, have been developed for malignant disorders at high doses [7]. Recently, these compounds have shown promise in lower doses as potent and safe anti-inflammatory drugs in autoimmune diseases such as systemic-onset juvenile idiopathic arthritis [8].

HDACs are grouped into class I (HDAC1, $-2,-3$ and -8 ), class IIa (HDAC4, $-5,-7$ and -9 ), class IIb (HDAC6 and -10) and class IV HDACs (HDAC11) [9]. Class I and IIb HDACs are ubiquitously produced whereas class IIa and IV are more tissue restricted. Among the class I HDACs, HDAC1, -2 and -8 were believed to reside in the nucleus whereas HDAC3 is also found in the cytoplasm [9]. However, recently the cytosolic localisation of HDAC1 and -2 has been demonstrated under pathologic conditions [10] and during development [11]. Class II and IV HDACs shuttle between the nucleus and cytoplasm [9].

All 11 classical HDACs are found in pancreatic beta cells in vitro [12], and beta cell levels of these enzymes are differentially regulated by exposure to IL-1 and IFN $\gamma$, key pro-inflammatory cytokines involved in inflammatory beta cell failure and destruction in type 1 diabetes [13]. HDAC inhibitors (HDACi) prevent cytokine-induced beta cell demise in vitro and in animal models of type 1 diabetes [14, 15]. However, it is unknown which HDACs mediate inflammatory beta cell destruction in cytokine-exposed beta cells and how HDAC1-3 are regulated in the islets of patients with type 1 diabetes. Here we used pharmacological inhibitors and small hairpin RNA (shRNA)-mediated transient and stable knock-down to reveal which HDACs mediate inflammatory beta cell damage, and we also studied human pancreatic sections to understand the regulation of these HDACs in children with recent-onset type 1 diabetes.

\section{Methods}

Cytokines and HDACi Mouse IL-1 $\beta$ was from BD Pharmingen (Erembodegem, Belgium) and rat IFN $\gamma$ from R\&D Systems (Oxford, UK). ITF compounds were provided in coded form from Italfarmaco (Cinisello Balsamo, Italy). LAQ842, vorinostat and PCI24781 were from Selleck Chemicals (Houston, TX, USA), Helminthosporium carbonum (HC) toxin and PCI34051 were from Sigma-Aldrich 
(St Louis, MO, USA) and CI-994 was from Exclusive Chemistry Ltd (Obninsk, Russia).

Plasmids pMD2.G (12259) and psPAX2 (12260) were from Tronolab (Lausanne, Switzerland). The empty vector (pLKO.1) was from Sigma-Aldrich. The vector plasmids were from Sigma-Aldrich (HDAC1: TRCN0000229438, TRCN0000039400) and GeneCopoeia (Rockville, MD, USA) (HDAC2: RSH054257-1, RSH054257-4; HDAC3: RSH051653-4, RSH051653-2).

Cells INS-1 cells were a gift from C. Wollheim, Department of Cell Physiology and Metabolism, University Medical Center, Geneva, Switzerland [16], and were maintained in complete medium as described [12]. At the initiation of experiments, medium was changed, and HDACi or vehicle were added $1 \mathrm{~h}$ before cytokine exposure.

The 293 FT cells (Invitrogen, Taastrup, Denmark) were maintained and prepared for transfection in accordance with the supplier's manual. HTB-9 cells (ATCC, Manassas, VA, USA) were maintained in complete medium without $\beta$ mercaptoethanol and cultured at $37^{\circ} \mathrm{C}$ in a humidified atmosphere containing $5 \% \mathrm{CO}_{2}$.

Lentivirus production and transduction Plasmids were transformed (C7373-03, Invitrogen), amplified and purified (Fischer Scientific, Slangerup, Denmark). Virus was generated by cotransfection of HEK293 cells with the vector plasmid, psPAX2 plasmid and pMD2.g plasmid using Lipofectamine 2000 (Invitrogen). After $72 \mathrm{~h}$, virus was harvested by ultracentrifugation of the supernatant fraction at $50,000 \mathrm{~g}$ for $2 \mathrm{~h}$ and the pellet was then resuspended in medium. Titres were determined in HT1080 cells (Invitrogen) according to the supplier's manual. Stable INS-1 cells were generated by seeding one million cells in $2 \mathrm{ml}$ medium. After 2 days, medium was removed and $800 \mu \mathrm{l}$ viruscontaining medium added (multiplicity of infection [MOI] $>10$ ). Medium was replaced with $2 \mathrm{ml}$ complete medium $6 \mathrm{~h}$ after transduction. Transduced cells were selected for using $1.5 \mu \mathrm{g} / \mathrm{ml}$ puromycin (Sigma, Poole, UK) for 2 to 3 weeks.

Rat islet isolation, preculture and culture Primary neonatal rat islets were isolated from outbred Wistar rats (Taconic, Ejby, Denmark), 3 to 6 days old, as previously described [17] and cultured as previously reported [12]. These experiments were approved by the local veterinary ethics committee.

Rat islet dispersion and lentiviral transduction Precultured neonatal rat islets were trypsinised to single cells. Fifty thousand (cell death ELISA) or 100,000 (RNA extraction) single cells were seeded in 48 or 12 wells pre-coated with extracellular matrix $(\mathrm{ECM})$, and maintained in $0.5 / 1.5 \mathrm{ml}$
RPMI 1640 (Gibco, Taastrup, Denmark), supplemented with $2 \%$ human serum (Bio Whitaker, Basel, Switzerland), $11.1 \mathrm{mmol} / 1$ glucose, $100 \mathrm{U} / \mathrm{ml}$ penicillin and $100 \mu \mathrm{g} / \mathrm{ml}$ streptomycin. Dispersed cells were cultured at $37^{\circ} \mathrm{C}$ in a humidified atmosphere containing $5 \% \mathrm{CO}_{2}$. After $48 \mathrm{~h}$ medium was removed and replaced with $150 \mu \mathrm{l}$ (48 wells)/ $250 \mu \mathrm{l}(12$ wells) medium containing Lentivirus $(\mathrm{MOI}=10)$. Virus-containing medium was replaced with $0.5 \mathrm{ml}(48$ wells) $/ 1.5 \mathrm{ml}$ (12 wells) medium after $6 \mathrm{~h}$. Cytokines were added $72 \mathrm{~h}$ after virus transduction.

ECM HTB-9 cells were grown for 2 days beyond $100 \%$ confluency followed by one wash with water. Coated wells containing water were stored at $4^{\circ} \mathrm{C}$. Wells were washed once before use.

$I C_{50}$ activity assay Enzyme activity was measured in duplicate in 384-well plates using the HDAC-Glo I/II Assay (Promega, Madison, WI, USA). For $\mathrm{IC}_{50}$ curves, dilution series of ten different compound concentrations were made in fourfold steps in DMSO. The series were diluted ten times in assay buffer and $5 \mu \mathrm{l}$ compound was mixed with $5 \mu$ l enzyme followed by $10 \mu \mathrm{l}$ HDAC-Glo Reagent containing substrate. The $\mathrm{IC}_{50}$ for all compounds was determined with seven HDACs (BPS Bioscience, San Diego, CA, USA) using the following concentrations: $0.00625 \mathrm{ng} / \mu \mathrm{HDAC} ; 0.0030 \mathrm{ng} /$ $\mu 1$ HDAC2; $0.00625 \mathrm{ng} / \mu \mathrm{l}$ HDAC3; $0.12 \mathrm{ng} / \mu \mathrm{l}$ HDAC6; $0.10 \mathrm{ng} / \mu 1 \mathrm{HDAC} 8 ; 0.61 \mathrm{ng} / \mu 1 \mathrm{HDAC} 10 ; 3 \mathrm{ng} / \mu \mathrm{l}$ HDAC11. Enzymes were diluted in assay buffer.

Assay Z-factors were: HDAC1, 0.84; HDAC2, 0.79; HDAC3, 0.86; HDAC6, 0.74; HDAC8, 0.84; HDAC10, 0.82 ; HDAC11, 0.80. All compounds were tested for inhibition of assay components according to assay protocol. Luminescence signal was measured with BioTek Synergy 4 (BioTek, Winooski, VT, USA) after 40 min.

Cell viability An arbitrary cell index reflecting real-time attachment, morphology and proliferation of INS-1 cells was monitored using the xCELLigence RTCA SP (Roche Diagnostics, Mannheim, Germany) platform. E-plates (96 wells) were pre-coated with poly-D-lysine (Sigma). Fifty microlitres of medium was added per well for background impedance measurement before the addition of 20,000 INS- 1 cells in $20 \mu \mathrm{l}$ medium followed by $30 \mathrm{~min}$ incubation at room temperature. The E-plate was inserted into the RTCA and placed at $37^{\circ} \mathrm{C}$ with $5 \% \mathrm{CO}_{2}$. After $24 \mathrm{~h}, \mathrm{HDACi}$ were added in duplicate in $30 \mu \mathrm{l}$ medium. After $1 \mathrm{~h}$, cytokines or vehicle were added. Impedance was measured hourly and the AUC of the $48-72 \mathrm{~h}$ interval was calculated. Results are depicted as percentage rescue of cells treated with cytokine alone.

Cell death detection by ELISA Aliquots of 50,000 stably transduced INS-1 cells or 50,000 dispersed rat islet cells 
were seeded in 48 -well plates containing $0.5 \mathrm{ml}$ medium. Cytokine-induced apoptosis was determined as previously described [12].

Real-time PCR One million wild-type or stably transduced INS-1 cells, or 100,000 dispersed rat islet cells, were seeded in six-well plates in $2 \mathrm{ml}$ complete medium or 12-well plates in $1.5 \mathrm{ml}$ medium. RNA purification, cDNA synthesis and real-time PCR were performed as previously described [12]. TaqMan probes were as follows: Hprt1: Rn01318745_g1; iNos (also known as Nos2): Rn00561646_m1; Ill $\alpha$ (also known as Illa): Rn00566700_m1; Il1 $\beta$ (also known as Illb): Rn99999009_m1; Cxcl2: Rn00586403_m1; Tnfo (also known as Tnf): Rn99999017_m1; Cxcl10: Rn00594648_m1.

Insulin assay Fifty thousand stably transduced INS-1 cells or 50,000 dispersed rat islet cells were seeded in 48-well plates containing $0.5 \mathrm{ml}$ medium with $11 \mathrm{mmol} / 1$ glucose. Cells were exposed to cytokines and accumulated insulin was measured as previously described [12].

Chromatin immunoprecipitation assay For the chromatin immunoprecipitation (ChIP) assay, 5,000,000 stably transduced INS-1 cells were exposed to cytokines. At the indicated time points DNA/protein was crosslinked. Lysis buffer was added to cells which were then lifted and transferred to Eppendorf tubes. Samples were sonicated at high intensity for $10 \mathrm{~min}$ in pulses of $30 \mathrm{~s}$. p65 was immunoprecipitated as previously described [18] with $5 \mu \mathrm{g}$ p65 antibody (sc372-X; Santa Cruz, CA, USA). Immunoprecipitated chromatin was extracted, and the presence of specific DNA co-precipitated with $\mathrm{p} 65$ was determined by real-time PCR targeting a proximal and distal p65-binding site in the iNos promoter (see Electronic supplementary material [ESM] Table 1 for primers). Background was assessed by the average signal from two no-gene controls: beta-globin and 1q32A (see ESM Table 1 for primers). AUC was calculated and background signal was subtracted.

Pancreas specimens Five human pancreases removed at autopsy from patients who had recent-onset type 1 diabetes were selected randomly from a previously described collection [19]. Specimens had been fixed in buffered formalin, unbuffered formol saline or Bouin's fixative, and they were all paraffin embedded. The selected cohort from which pancreases were obtained consisted of patients with a mean \pm SD age of $5.5 \pm 1.9$ years (range $1-11$ years). Controls were five normal pancreases from individuals age-matched to the patients with type 1 diabetes, such that they had a mean \pm SD age of $6.0 \pm 1.6$ years (range 2-10 years; see ESM Table 2). These investigations were approved by the responsible ethics committees.
Immunohistochemistry Serial sections $(4 \mu \mathrm{m})$ were cut from each pancreas and mounted on glass slides coated in (3-aminopropyl)-triethoxysilane (Sigma). Sections were processed and labelled using a standard immunoperoxidase technique for paraffin sections. HDAC antigens were unmasked by heat-induced epitope retrieval in $10 \mathrm{mmol} /$ 1 citrate buffer $\mathrm{pH}$ 6.0. Primary antibodies (Abcam, Cambridge, UK) were applied for $1 \mathrm{~h}$ at room temperature, with the exception of anti-HDAC3, which was incubated overnight at $4{ }^{\circ} \mathrm{C}$. Dako REAL Envision Detection System (Dako, Cambridge, UK) was used for antigen detection. An arbitrary scoring system was developed to assess the intensity of staining and the slides were examined and scored independently by two observers. The staining intensity for each of three islets in each case was recorded and mean values were calculated.

Immunofluorescence To examine the islet cell subtypes producing HDAC isoforms and to confirm their cellular localisation, double immunofluorescence staining was performed. Antisera with specificity for HDAC1, -2 or -3 were incubated as described for the immunoperoxidase method above and were detected with goat Alexa Fluor 568-conjugated secondary antibody (Invitrogen, Paisley, UK). The sections were washed and stained with guineapig anti-insulin serum (Dako), detected with a goat antiguinea-pig secondary antibody conjugated with Alexa Fluor 488. DAPI $(1: 1,000$; Invitrogen) was included in the final secondary incubation. Sections were mounted in Vectashield hard-set mounting medium (Vector, Peterborough, UK) under glass coverslips. Sections were analysed using identical settings and captured using a Nikon Eclipse $80 i$ microscope (Nikon, Kingston upon Thames, UK) and images were overlaid using NIS-Elements BR 3.0 software (Nikon).

Statistical analysis Comparisons between groups were carried out either by paired $t$ test or by ANOVA followed by pre-planned post hoc tests.

\section{Results}

Cytokine-induced beta cell toxicity is mediated through class I HDACs We first screened 13 selective HDACi (Italfarmaco, Cinisello Balsamo, Italy) for their ability to preserve the viability of INS-1 cells during exposure to the cytokines IL- $1 \beta$ and IFN $\gamma$. The screening was performed using the realtime xCELLigence platform, which calculates a cell index, based on plate impedance, closely correlated with the cell adherence area and is thereby a surrogate of cell viability, as exemplified with compound ITF-J in Fig. 1a. 
a

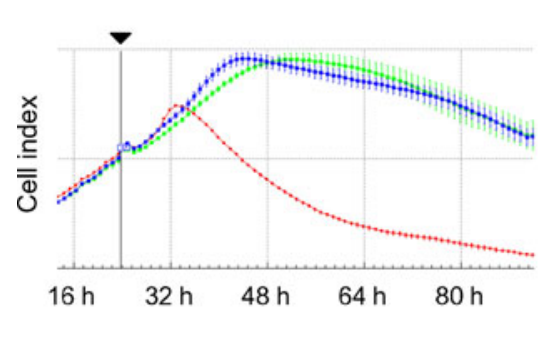

C

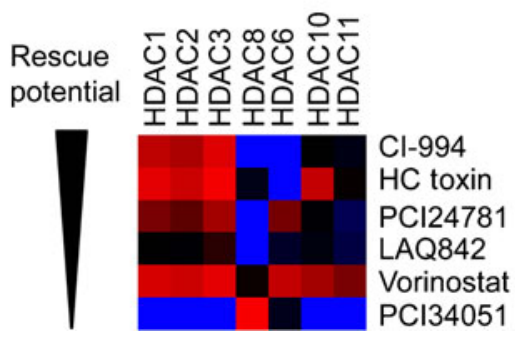

Fig. 1 Class I selective HDACi have the highest INS-1 rescue potential. INS-1 cells were monitored using the real-time xCELLigence system $\mathrm{h}$ and the impedance (cell adhesion) was measured as a surrogate of cell viability (cell index) as described in the Methods. (a) The impedance of duplicates of control (green line), cytokine-exposed (red line) and cytokine+ITF-J-exposed INS-1 cells (blue line) was followed from the start of exposure (indicated by the arrow). Heat maps of 13 different ITF HDAC inhibitor compounds (b) or six different

The most efficient compounds were class I, IIb and IV HDACi, whereas inhibitors of class IIa HDACs were ineffective (Fig. 1b, ESM Table 3). We next screened six commercially available HDACi (structures shown in ESM Fig. 1), namely HC toxin, CI-994, PCI24781, LAQ824, vorinostat and PCI34051, for which we determined the inhibitory activity on class I, IIb and IV HDACs. HC toxin, CI-994, PCI24781 and LAQ824 all protected against cytokine-induced beta cell death, whereas the HDAC8selective inhibitor PCI34051 showed no protection (Fig. 1c, ESM Table 4), a finding verified using two other HDAC8 selective inhibitors (ITF-T, Fig. 1, ESM Table 3, and data not shown). CI-994, a highly selective HDAC1-3 inhibitor, had the highest rescue efficacy. HC toxin, ranking second in rescue efficacy, did not inhibit HDAC6.

These results suggest that the class I HDACs HDAC1, -2 and/or -3 mediate cytokine-induced beta cell toxicity and these HDACs were thus chosen for further knock-down studies.

Knock-down of HDAC1, -2 or -3 decreases cytokine-induced iNos mRNA expression, but only knock-down of HDAC1 and -3 reduces cytokine-induced apoptosis We generated stable INS-1 clones deficient in HDAC1, -2 or -3 (ESM Fig. 2). As shown in ESM Fig. 2a-c there was a significant increase in the mRNA expression of both Hdacl and -3 in cells deficient in HDAC2; of note this did not translate into changes in protein levels. Deficiency of HDAC1 and -3

\section{b}

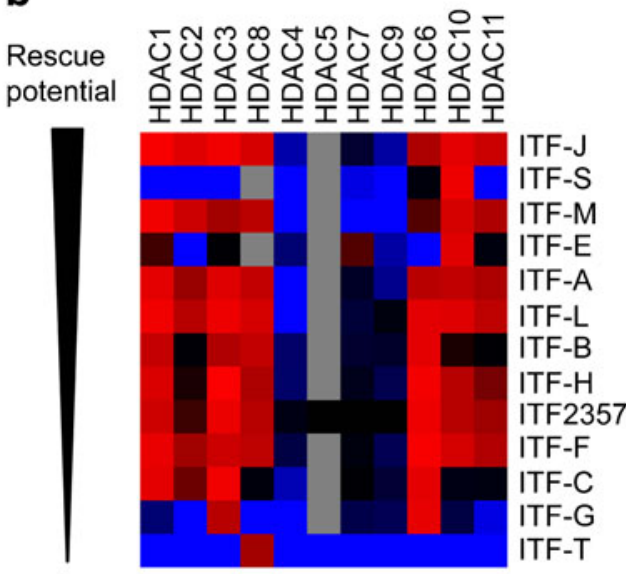

d

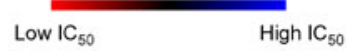

commercial HDAC inhibitor compounds (c) were made based on their $\mathrm{IC}_{50}$ values towards selected HDACs. The HDACi inhibitors are ranked after rescue potential according to ESM Tables 1, 2. (c) Values are corrected for differences in potency since they varied from 33.3 (CI-994) to 0.041 (LAQ824). (d) Colour code for both heat maps: low $\mathrm{IC}_{50}$ values coloured red, intermediate $\mathrm{IC}_{50}$ values coloured black and high $\mathrm{IC}_{50}$ values coloured blue; grey indicates undetermined $\mathrm{IC}_{50}$

significantly reduced the expression of Hdac2 mRNA (ESM Fig. 2b), while HDAC2 protein levels appeared upregulated in HDAC1-deficient cells (ESM Fig. 2d, f), in line with previous observations $[20,21]$. These data suggest interdependent regulation of HDAC expression, as previously observed with pharmacological inhibition [12].

HDAC inhibition blocks cytokine-induced inducible nitric oxide synthase (iNOS) production [12, 14, 22]. We therefore investigated whether a deficiency in HDAC1, -2 or -3 affected cytokine-mediated $i N o s$ induction in INS-1 cells. IL-1 $\beta$ and IFN $\gamma$ induced iNos mRNA by more than 140,000-fold in empty vector expressing cells (data not shown). Empty vector transduction did not affect cytokine-induced iNos mRNA production compared with wild-type INS-1 cells (Fig. 2a). In contrast each of the two shRNA constructs against Hdacl, -2 or -3 significantly reduced $i$ Nos mRNA expression.

The INS-1 clones with the most efficient knock-down were selected for further studies (ESM Fig. 2). Knock-down of HDAC1 and -3 significantly protected INS-1 cells from cytokine-induced apoptosis by $38 \%$ and $41 \%$, respectively (Fig. 2b, d), whereas knock-down of HDAC2 showed no effect (Fig. 2c).

Cytokine-induced toxicity is mediated by HDAC1 and -3, and knock-down of HDACl completely restores insulin secretion in dispersed primary rat islets To further investigate the effects of HDAC1-3 knock-down on cytokine-induced iNos 

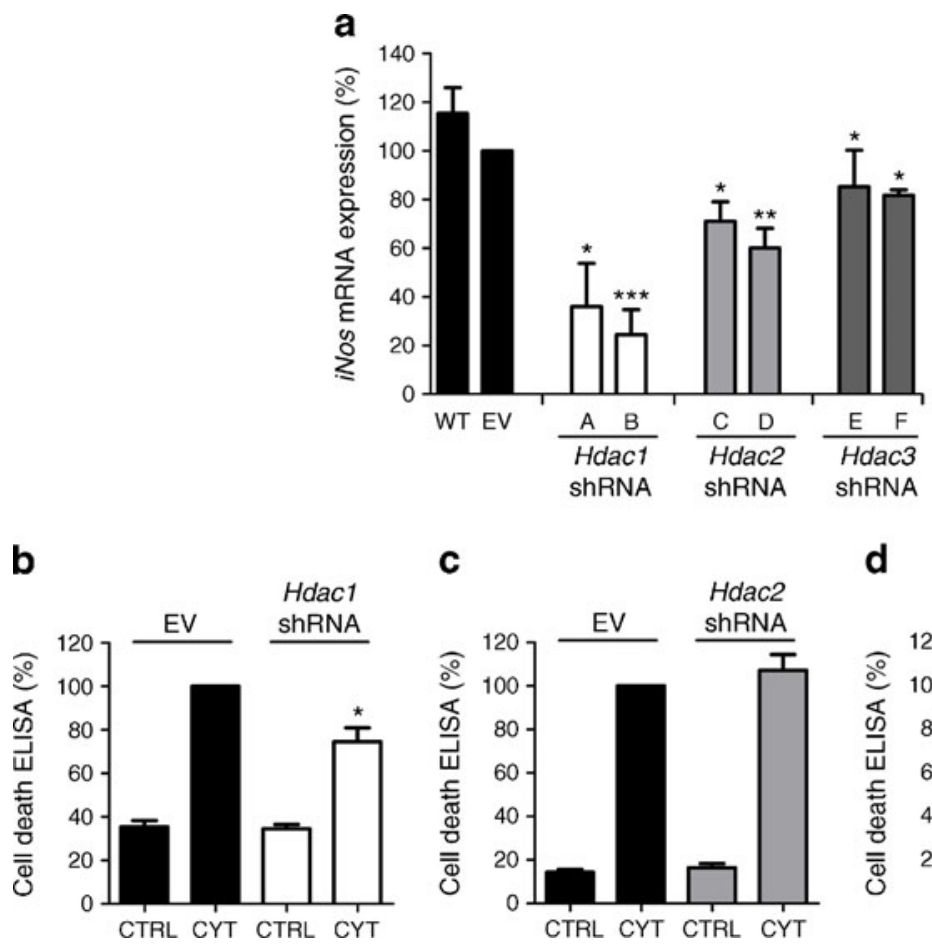

c

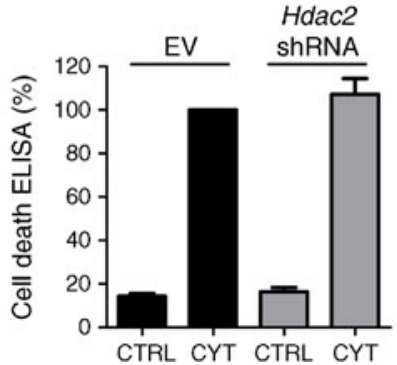

d

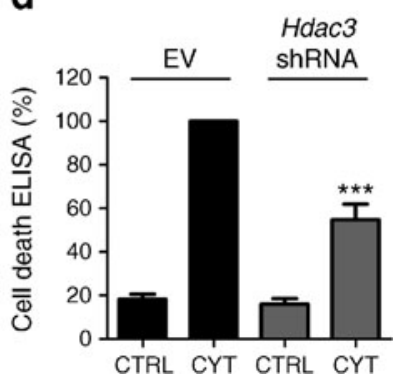

Fig. 2 Specific knock-down of HDAC1, -2 or -3 in INS-1 cells decreases cytokine-induced iNos mRNA expression, but only knockdown of HDAC1 and -3 reduces cytokine-induced apoptosis. (a) Wildtype (WT), empty vector (EV), Hdacl shRNA (A and B, white bars), Hdac2 shRNA (C and D, light grey bars) or Hdac3 shRNA (E and F, dark grey bars)-treated cells were cultured for $6 \mathrm{~h}$ in the presence of IL$1 \beta(150 \mathrm{pg} / \mathrm{ml})+\operatorname{IFN} \gamma(0.1 \mathrm{ng} / \mathrm{ml})$. Total RNA was isolated, cDNA generated by reverse transcription and expression of $i$ Nos quantified by real-time PCR. To correct for variation in input, data were normalised to the expression level of Hprt1. Data are presented as means + SEM compared with EV cells exposed to cytokines (black bar), $n=3-5$,

mRNA expression and cell death, we performed transient knock-down of HDAC1-3 in dispersed primary rat islet cells (ESM Fig. 2g-i). The interdependence of HDAC regulation observed in INS-1 cells was confirmed in dispersed islet cells as knock-down of HDAC1 decreased Hdac2 mRNA expression and HDAC2 knockdown increased Hdac3 mRNA expression. Cytokineinduced $i$ Nos expression was reduced by HDAC1 and -3 knock-down, while HDAC2 knock-down had no effect (Fig. 3a, ESM Fig. 3). Importantly, knock-down of HDAC1 and -3 reduced cytokine-induced apoptosis by $41 \%$ and $42 \%$, respectively (Fig. 3 b). Of further interest, HDAC1 knock-down significantly restored accumulated insulin release, whereas knocking down HDAC2 or -3 was ineffective (Fig. 3c).

Differential roles of $H D A C 1-3$ on $N F \kappa B$ Since the protective mechanism of HDAC inhibition on cytokine-induced toxicity is not mediated through upregulation of the antiapoptotic genes Socs1, Socs3 or Sirt1 [23], we sought to
$* p<0.05, * * p<0.01, * * * p<0.001$ (ANOVA, pre-planned post hoc test). (b-d) Stable Hdac1 shRNA (white bars) (b); Hdac2 shRNA (light grey bars) (c) or Hdac3 shRNA (dark grey bars)-expressing INS-1 cells (d) were exposed to IL-1 $\beta(150 \mathrm{pg} / \mathrm{ml})+$ IFN $\gamma$ $(0.1 \mathrm{ng} / \mathrm{ml})(\mathrm{CYT})$ or vehicle (CTRL) for $24 \mathrm{~h}$. Stable INS-1 cells expressing an EV were used as control. The quantity of DNA-histone complexes present in the cytoplasm (i.e. apoptosis) was determined by cell death detection ELISA. Data are presented as means + SEM compared with EV-expressing INS-1 cells exposed to cytokines $(n=5) ; * p<0.05, * * * p<0.001$ vs cytokine-exposed EV INS-1 cells (ANOVA, pre-planned post hoc test)

investigate the effect of knocking down HDAC1-3 on NFKB binding to the $i N o s$ proximal and distal promoter regions. Knock-down of HDAC3 significantly reduced p65 binding (Fig. 4), whereas knock-down of HDAC1 and -2 did not significantly affect p65 binding (although a trend was observed for HDAC2 knock-down). An NFKB reporter assay further indicated a role for HDAC2 and -3 in the upregulation of NFKB activity (ESM Fig. 4). These findings suggest that the protective effects of HDAC1 and -3 knock-down are mediated by different mechanisms.

HDAC1, -2 or -3 knock-down differentially alters cytokineinduced expression of pro-inflammatory mediators We recently showed that the broad HDAC inhibitor givinostat decreased cytokine-induced beta cell expression of several pro-inflammatory mediators (Ill $\alpha, \operatorname{Ill} \beta, \operatorname{Tnf} \alpha$ and $\mathrm{Cxcl} 2)$ [23].

Since only HDAC3-mediated cytokine-induced iNos expression depended on NF $\kappa \mathrm{B}-\mathrm{DNA}$ binding, we wished to 


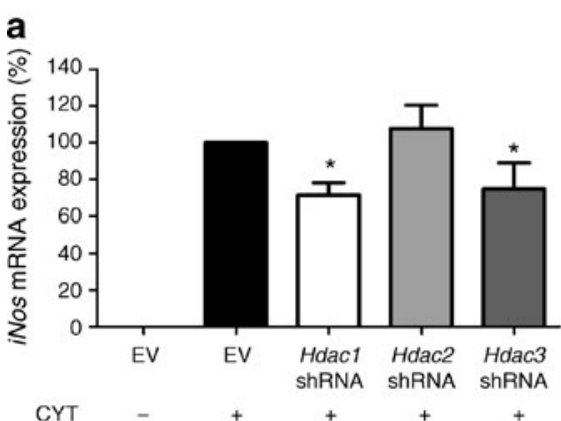

Fig. 3 Knock-down of HDAC1 or -3 , but not HDAC2, protects dispersed rat islets against cytokine-induced apoptosis and only HDAC1 knock-down restores insulin secretion. Dispersed rat islets were transduced with specific $\mathrm{HDAC} 1 / 2 / 3$ or an empty vector (EV) shRNA for $72 \mathrm{~h}$ before IL-1 $\beta(500 \mathrm{pg} / \mathrm{ml})+\operatorname{IFN} \gamma(1 \mathrm{ng} / \mathrm{ml})$ exposure CYT or vehicle for $6 \mathrm{~h}$ (a) or $20 \mathrm{~h}$ (b and c). (a) Transduced cells were cultured in the presence or absence of cytokines. Total RNA was isolated, cDNA generated by reverse transcription and expression of $i$ Nos quantified by real-time PCR. To correct for variation in input, data were normalised to the expression level of Hprt1. Data are presented as means+SEM compared with EV cells exposed to cytokines (black bar),

test whether a similar differential effect of HDAC1-3 could be demonstrated for other inflammatory mediators typically regulated by $\mathrm{NFKB}$ activity.

Cytokine-induced INS-1 cell expression of $I l 1 \alpha, I l 1 \beta$, Tnfo and Cxcl2 (Fig. 5 a-d) was significantly reduced by HDAC1 knock-down. Interestingly, HDAC2 knock-down reduced the expression of $I l 1 \alpha, I l 1 \beta$ and Tnf $\alpha$ (Fig. 5 a-c) but not of Cxcl2 (Fig. 5d). Notably, HDAC3 knock-down increased cytokine-mediated induction of $I l l \alpha$ and $\operatorname{Tn} f \alpha$ (Fig. 5a, c), whereas $I l 1 \beta$ and $C x c l 2$ (Fig. 5b, d) induction was unaffected. The basal expression of $\mathrm{Cxcl} 2$ was reduced by HDAC 1 knock-down, increased by HDAC 2 knockdown and unaffected by HDAC3 knock-down (Fig. 5e). Basal expression of the other pro-inflammatory mediators was undetectable. These data further support non-redundant functions of class I HDACs on inflammatory gene expression in insulin-producing cells.

\section{Islet HDAC1-3 expression in pancreatic sections from chil-} dren without diabetes and children with recent-onset type 1 diabetes $\mathrm{HDAC} 1-3$ were detected by immunohistochemistry in pancreas sections from five children without diabetes (Fig. 6a, case details in ESM Table 2), and prevalence was considerably higher in the islet cells than in the surrounding exocrine tissue. Immunofluorescence analysis revealed that HDAC1 staining was mainly cytosolic in islet endocrine cells and that those cells stained most intensely were not immunopositive for either glucagon or insulin (data not shown). Insulinpositive cells displayed a very low level of HDAC1 whereas HDAC2 and HDAC3 were each labelled more intensely in insulin-positive cells and were localised principally within the nucleus. This suggests that human beta cells contain all three HDAC isoforms but that they are differentially localised.

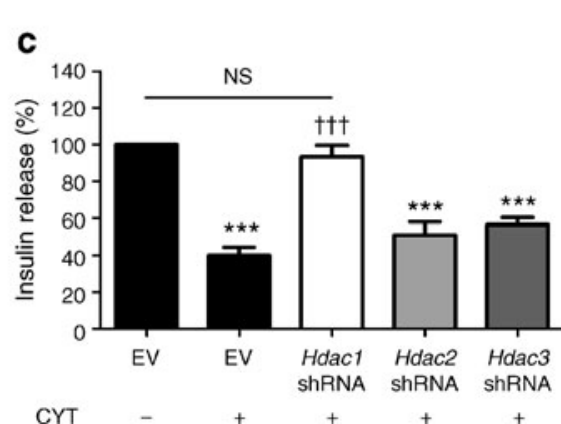

$n=4, * p<0.05$, (ANOVA, pre-planned post hoc test). (b) The quantity of DNA-histone complexes present in the cytoplasm (i.e. apoptosis) was determined by cell death detection ELISA. Data are presented as means + SEM compared with EV exposed to cytokines $(n=3) ;{ }^{*} p<$ 0.05 , vs cytokine-exposed EV cells (ANOVA, pre-planned post hoc test). (c) Medium was collected and insulin content measured by competitive ELISA. Data are presented as means + SEM compared with vehicle-exposed EV $(n=3) ; * * * p<0.001$, vs vehicle-exposed EV cells, ${ }^{\dagger \dagger} p<0.001$, vs cytokine-exposed EV cells (ANOVA, preplanned post hoc test)

The islet production of HDAC1 was markedly increased in pancreas sections from five age-matched patients with recent-onset type 1 diabetes (Fig. 6b). Dual immunofluorescence labelling demonstrated that this enzyme was upregulated in the beta cells in type 1 diabetes (Fig. 6d) since it was barely detectable by this method in the insulin-positive cells of controls without diabetes (Fig. 6c). Some immune cells also stained positively for HDAC1 in the inflamed islets of patients with type 1 diabetes, although with a nuclear distribution (Fig. 6d, indicated by arrows). Labelling of HDAC2 and HDAC 3 was significantly reduced in the islets of patients with recent-onset type 1 diabetes compared with controls (Fig. 6b). This reduction was not due to the loss of insulin-containing beta cells since staining was also reduced in those islets that retained insulin positivity (Fig. 6d).

\section{Discussion}

There is accumulating evidence that HDACs may be novel pharmacological targets in immune-mediated beta cell destruction leading to type 1 diabetes. Thus, HDACi, such as givinostat, vorinostat and trichostatin A, reduce cytokineinduced beta cell toxicity and restore insulin secretion in vitro and in vivo [12, 14, 15, 22, 24]. However, all HDACi in current clinical use are non-selective, and it is not known which of the 11 HDACs produced by the beta cell [12] mediate the toxic effects of cytokines. Using $19 \mathrm{HDACi}$ with selectivity for different HDACs we identified HDAC1, -2 and -3 as potential cytokine-regulated targets in beta cells. Specific knock-down of HDAC1 and -3, but not HDAC2, reduced cytokine-induced apoptosis and $i N o s$ expression whereas only knock-down of HDAC3 altered the binding of NFKB to the 

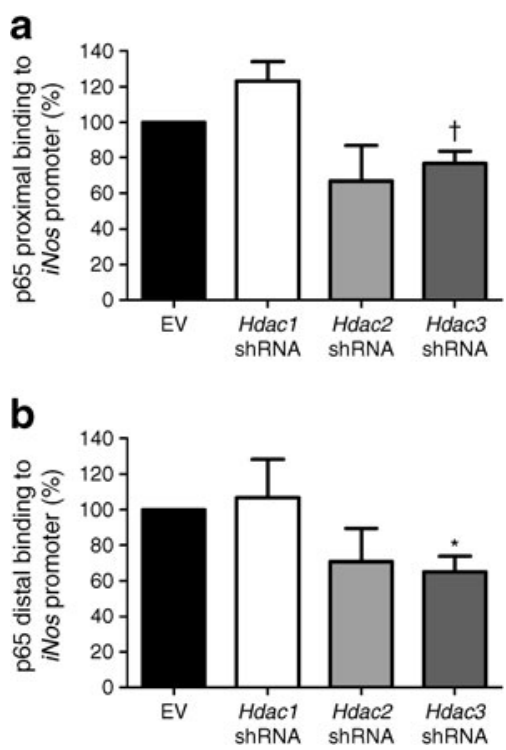

Fig. 4 p65 binding to genomic iNos promoter is attenuated by knockdown of HDAC3. Stable Hdac1, Hdac2 or Hdac3 shRNA-expressing INS- 1 cells were exposed to a mixture of cytokines IL-1 $\beta(600 \mathrm{pg} / \mathrm{ml})$ and $\operatorname{IFN} \gamma(0.1 \mathrm{ng} / \mathrm{ml})$ for $15,30,60,90$ and $120 \mathrm{~min}$ and cells were lysed. Stable INS-1 cells expressing an empty vector (EV) were used as control. p65 binding to proximal (a) and distal (b) $\mathrm{kB}$ sites in the iNos promoter in genomic DNA was assessed by ChIP and quantified by real-time PCR. AUCs were calculated after subtracting background signal and normalised to EV. Data are presented as means + SEM compared with EV cells exposed to cytokines (black bar), $n=4,{ }^{*} p<0.05$ vs EV (Student's paired $t$ test); ${ }^{\dagger} p=0.07$ vs EV (Student's paired $t$ test)

iNos promoter. Furthermore, HDAC1, -2 and -3 differentially regulated beta cell expression of four cytokineinduced pro-inflammatory cytokines. Interestingly, only knock-down of HDAC1 restored the inhibited insulin secretion in primary cells exposed to cytokines. Finally, we examined the levels of HDAC1-3 in pancreases from patients with type 1 diabetes and from control participants and found HDAC1 to be upregulated and located in the beta cell cytosol in diabetic children whereas HDAC2 and 3 were both downregulated and confined to the nucleus.

The striking consistency between the experiments using HDACi and lentiviral knock-down of selected HDACs in both an insulin-producing cell line and dispersed primary rat islet cells strengthen these observations. First, the important protective effect of knocking down HDAC1 and -3 was demonstrated in both models. Second, the reduction of cytokineinduced beta cell apoptosis in the cell line and the primary cells was identical for both HDAC1 (38\% and 40\%, respectively) and -3 ( $41 \%$ and $42 \%$, respectively). Only the effect of knocking down HDAC2 on cytokine-induced INS-1 cell iNos expression was not confirmed in primary islet cells. This may result from differences in differentiation between the two model systems as described previously $[25,26]$.

A compelling effect of HDAC inhibition is the ability to restore accumulated insulin release in the beta cell $[12,14]$, an action mimicked by HDAC1 knock-down in primary islets cells. Indeed, at low glucose concentrations HDAC1 interacts with PDX-1, a transcription factor decisive for the maintenance of the insulin-secreting phenotype of pancreatic beta cells, and this interaction reduces insulin gene expression in insulin-producing MIN6 cells [27, 28].

An intriguing finding was that HDAC1 and -2 , but not HDAC3, deficiency reduced cytokine-induced expression of $I l 1 \alpha, I l 1 \beta$ and $T n f \alpha$, whereas HDAC3, and to some extent HDAC2, deficiency reduced NFKB-iNos promoter binding. These data illustrate three important points: (1) HDAC1, -2 and -3 regulate constitutive and cytokine-induced gene expression in a distinct manner; (2) cytokine-induced beta cell mRNA expression of $I l 1 \alpha, I l 1 \beta$ and Tnf $\alpha$ is dispensable for cytokine-induced beta cell apoptosis; (3) HDAC1 regulates pro-inflammatory gene expression, including $i N o s$, independently of NF KB-DNA-binding, probably by interfering with NFKB signalling at a more proximal level (e.g. by affecting co-factor binding to $\mathrm{p} 65$ or by targeting alternative transcription factors) [29]. Thus, HDAC1-3 all form complexes with the p65 subunit and regulate NFKB-mediated genes in non-beta cells, but only HDAC3 actually deacetylates p65 $[6,30,31]$.

Despite considerable homology between HDAC1 and -2 in terms of structure and protein-interaction partners [9], we found distinct effects of HDAC1 or -2 deficiency on basaland cytokine-induced gene expression, apoptosis and insulin secretion. Similarly, HDAC1 and -2 differentially regulate stem cell differentiation [32], extracellular signal-related kinase (ERK)/mitogen-activated protein kinase (MAPK) apoptotic cytokine signalling [21] and TGF- $\beta$-accelerated diabetic nephropathy [33].

Importantly, HDACs also play a vital role in the development of the endocrine pancreas. Thus inhibition of class IIa HDACs increases the beta/delta cell mass, whereas inhibition of class I HDACs supports alpha/PP cell expansion [34, 35]. These observations warrant future development of class IIa selective HDACi for beta cell differentiation-based therapies and HDAC1- and HDAC3-selective HDACi in antiinflammatory-based strategies.

We have previously reported that cytokines downregulate HDAC $1-3$ expression in primary rat islets and HDAC 1 and -2 in human islets in vitro [12]. Here we show that HDAC2 and 3 levels are downregulated in islet cells in children with recent-onset type 1 diabetes, possibly reflecting a secondary protective response to inflammatory assault, although it should be recognised that their activity may well be higher compared with healthy individuals. Hence, selective HDACi hold therapeutic promise both before and after disease onset.

Interestingly, not only the production but also the cellular compartmentalisation determines the pathologic actions of HDAC1. Thus, through a newly identified Nuclear Export Signal [10] HDAC1 is translocated from the nucleus to the 


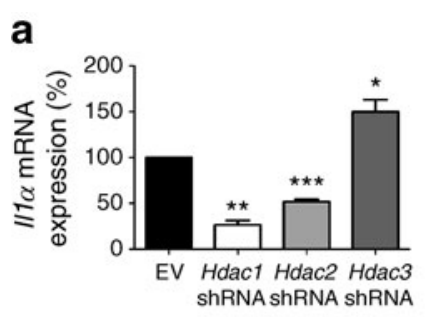

d

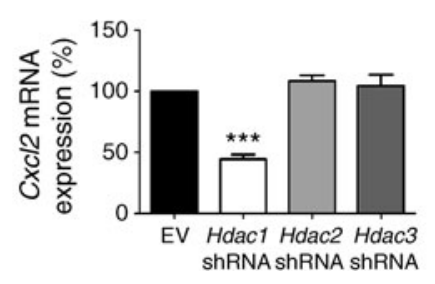

Fig. 5 Knock-down of HDAC1, -2 or -3 differentially regulates basal and cytokine-induced expression of several pro-inflammatory cytokines. (a-e) Stable Hdacl, Hdac2 or Hdac3 shRNA-expressing INS1 cells were exposed to IL-1 $\beta(150 \mathrm{pg} / \mathrm{ml})+\operatorname{IFN} \gamma(0.1 \mathrm{ng} / \mathrm{ml})$ or vehicle for $6 \mathrm{~h}$. Stable INS-1 cells expressing an empty vector (EV) were used as control. Total RNA was isolated, cDNA was generated by reverse transcription and expression of $I l 1 \alpha(\mathbf{a}), I l 1 \beta(\mathbf{b}), \operatorname{Tn} f \alpha(\mathbf{c})$ and

cytoplasm in human neurons and impairs axonal transport, restored by preventing HDAC1 shuttling. Therefore, the cytoplasmic localisation of HDAC1 observed in the islets of patients with type 1 diabetes may be a pathological response contributing to pro-apoptotic signalling and impairment of insulin secretion.

Our study further strengthens the notion that HDACi should not only be considered as antineoplastic therapies, but also as potential treatments for inflammatory disorders such as diabetes as reviewed elsewhere [36, 37]. Similar anti-inflammatory properties have been demonstrated in lipopolysaccharidestimulated human peripheral blood mononuclear cells [38], an animal model of rheumatoid arthritis [39] and patients suffering from systemic-onset juvenile idiopathic arthritis (SOJIA) [8]. Importantly, a class I HDAC inhibitor was superior as an anti-rheumatic therapy compared with a paninhibitor, further underscoring the role of class I HDACs in inflammatory disorders [39]. In addition, the HDAC inhibitor sodium phenylbutyrate (PBA) increased the insulin sensitivity in NEFA- and triacylglycerol-infused patients [40], confirming earlier reports showing that PBA reduces endoplasmic reticulum (ER) stress and restores glucose homoeostasis in an animal model of type 2 diabetes mellitus [41]. PBA is also a chemical chaperone that protects cells by reducing ER stress. Alternatively the protective effect of PBA could be exerted by inhibiting the deleterious effects of cytokines produced by macrophages in response to NEFAinduced beta cell macrophage chemoattractant production [42]. HDAC knock-down may reveal the mechanisms by which PBA ameliorates NEFA-induced beta cell demise.
C
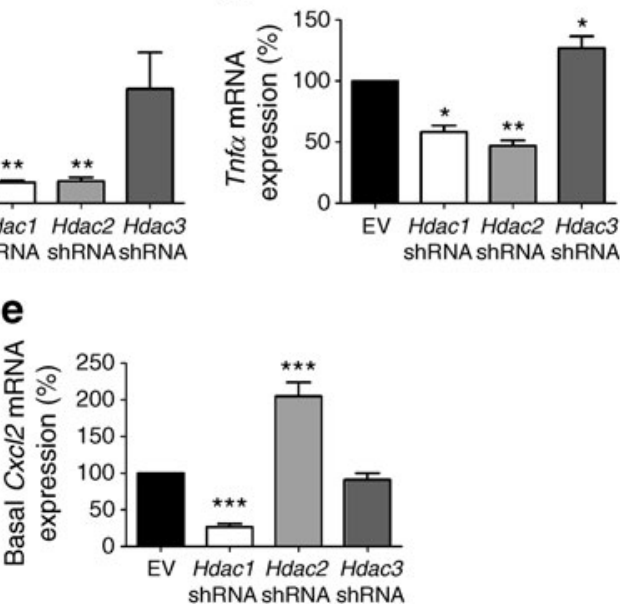

Cxcl2 (d and e) was quantified by real-time PCR. To correct for variation in input, data were normalised to the expression level of Hprt1. Data are presented as means + SEM compared with EV cells exposed to cytokines (black bar), $n=3-5,{ }^{*} p<0.05, * * p<0.01, * * * p<$ 0.001 (a-c: Student's paired $t$ test, $\mathbf{d}$ and e: ANOVA, pre-planned post hoc test)

In conclusion HDAC1, -2 and -3 have distinct pathologic functions in the beta cell. In vitro, HDAC1 and -3 are each required for cytokine-induced beta cell death, whereas HDAC1 also selectively impairs insulin secretion. Conversely only HDAC3 appears to be involved in cytokineinduced NFKB DNA binding. In the beta cells of children with type 1 diabetes, HDAC1 was upregulated and was localised in the cytosol, whereas HDAC2 and -3 were localised to the nucleus and were downregulated. These findings suggest that targeting HDAC1 and HDAC3 should provide optimal protection of beta cell mass and function in clinical islet transplantation and in patients with recent-onset type 1 diabetes.

Acknowledgements We thank D. Trono (Tronolab, School of Life Sciences, École Polytechnique Fédérale de Lausanne, Switzerland) for providing the envelope, package and empty vector plasmids. G. K. Singh (Center for Medical Research Methodology, Department of Biomedical Sciences, Faculty of Health and Medical Sciences, University of Copenhagen, Copenhagen, Denmark) is thanked for technical assistance.

Funding This work was supported by JDRF (grant no. 26-2008893), NIH (AI-15614 and AR-45584), The IL-1 Foundation, a University of Copenhagen career PhD fellowship, a Novo Nordisk STAR Postdoc fellowship, Diabetes Research and Wellness Foundation and the Novo Nordisk Foundation.

Duality of interest P. Mascagni and A. Stevenazzi are employed by Italfarmaco. All other authors declare that there is no duality of interest associated with this manuscript. 
a

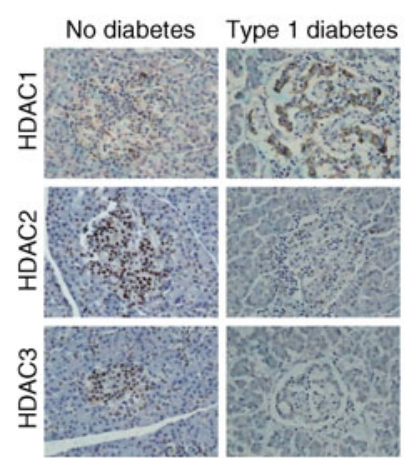

b

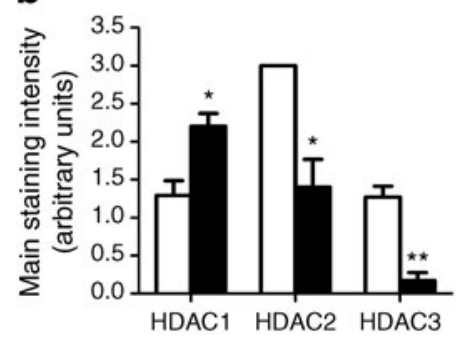

C
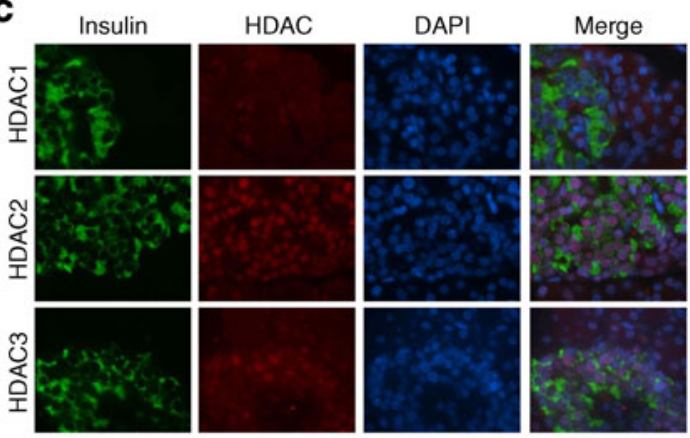

d
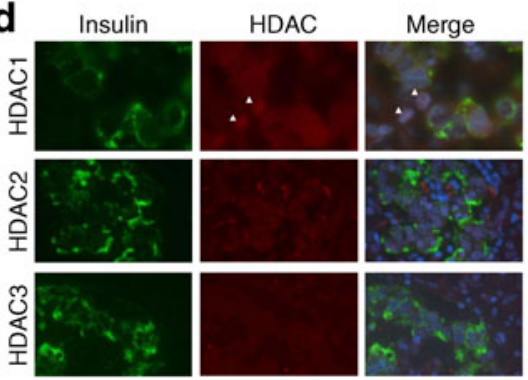

Fig. 6 HDAC expression in pancreatic islets from non-diabetic subjects and patients with recent-onset type 1 diabetes. (a) Photomicrographs of representative islets from control without diabetes (C1) and patients with recent-onset type 1 diabetes (D4 [HDAC1 and -2] and D5 [HDAC3] in ESM Table 2) stained with antisera raised against HDAC1, -2 and -3 . (b) Each case was stained for the presence of HDAC $1,-2$ and -3 and three randomly selected islets separately, coded and scored for staining intensity by each of two independent observers. The mean islet staining intensity + SEM (arbitrary units) for control cases without diabetes (white bars) and patients with type 1 diabetes (black bars) is presented, $n=5,{ }^{*} p<0.05,{ }^{*} p<0.01$ vs control (Student's paired $t$ test). (c) Immunofluorescence labelling of HDAC1, -2 and -3 in a typical control pancreas $(\mathrm{C} 1)$. Immunostaining for insulin

Contribution statement ML, LGG, NGM and TMP planned and designed the study. CAD, AS and PM synthesised and performed HDAC activity assays of the ITF inhibitors. TS and JB carried out HDAC activity assays for the commercially available HDACi. SJR and NGM were responsible for histological analyses. All other experimental work was carried out by ML, DPC, MDN and MSD. ML and TMP wrote the first draft of the manuscript, which was commented on by all authors. ML, DPC, MSD and TMP edited the final manuscript. All authors approved the final version. TMP is the guarantor of this work and, as such, had full access to all the data in the study and takes responsibility for the integrity of the data and the accuracy of the data analysis.

\section{References}

1. The Canadian-European Randomized Control Trial Group (1988) Cyclosporin-induced remission of IDDM after early intervention. Association of $1 \mathrm{yr}$ of cyclosporin treatment with enhanced insulin secretion. Diabetes 37:1574-1582

2. Keymeulen B, Vandemeulebroucke E, Ziegler AG et al (2005) Insulin needs after CD3-antibody therapy in new-onset type 1 diabetes. N Engl J Med 352:2598-2608 (green), HDACs (red) and DAPI (blue) are shown, together with the overlaid images obtained for all three antisera. (d) Immunofluorescence labelling of patients D4 (HDAC1 and -2) and D5 (HDAC3) for insulin (green) and HDACs (red) is shown, together with overlaid images co-stained with DAPI. Upper panel: HDAC1 is observed in insulin-positive cells of a pancreas from a patient with type 1 diabetes (D4). The presence of HDAC1 in the nuclei of infiltrating immune cells is also demonstrated (indicated by arrows). Immunostaining also demonstrates a reduction in HDAC2 (D4) and -3 (D5) in beta cells from a pancreas from a patient with type 1 diabetes compared with control (c). Magnification in panels (c) and (d): $\times 1,000$ (with the exception of HDAC1, panel $\mathbf{d}, \times 2,000$ )

3. Pescovitz MD, Greenbaum CJ, Krause-Steinrauf H et al (2009) Rituximab, B-lymphocyte depletion, and preservation of beta-cell function. N Engl J Med 361:2143-2152

4. Berger JR, Houff SA, Major EO (2009) Monoclonal antibodies and progressive multifocal leukoencephalopathy. MAbs 1:583589

5. Choudhary C, Kumar C, Gnad F et al (2009) Lysine acetylation targets protein complexes and co-regulates major cellular functions. Science 325:834-840

6. Lf C, Fischle W, Verdin E, Greene WC (2001) Duration of nuclear NF-kappa B action regulated by reversible acetylation. Science 293:1653-1657

7. Federico M, Bagella L (2011) Histone deacetylase inhibitors in the treatment of hematological malignancies and solid tumors. J Biomed Biotechnol 2011:475641

8. Vojinovic J, Damjanov N, D'Urzo C et al (2011) Safety and efficacy of an oral histone deacetylase inhibitor in systemic-onset juvenile idiopathic arthritis. Arthritis Rheum 63:1452-1458

9. de Ruijter AJ, van Gennip AH, Caron HN, Kemp S, van Kuilenburg AB (2003) Histone deacetylases (HDACs): characterization of the classical HDAC family. Biochem J 370:737-749

10. Kim JY, Shen S, Dietz K et al (2010) HDAC1 nuclear export induced by pathological conditions is essential for the onset of axonal damage. Nat Neurosci 13:180-189 
11. Bakin RE, Jung MO (2008) HDAC2 cytoplasmic sequestration potentiates keratinocyte terminal differentiaiton. Open Cell Dev J 1:1-9

12. Lundh M, Christensen DP, Rasmussen DN et al (2010) Lysine deacetylases are produced in pancreatic beta cells and are differentially regulated by proinflammatory cytokines. Diabetologia 53:2569-2578

13. Eizirik DL, Mandrup-Poulsen T (2001) A choice of death - the signal-transduction of immune-mediated beta-cell apoptosis. Diabetologia 44:2115-2133

14. Larsen L, Tonnesen M, Ronn S et al (2007) Inhibition of histone deacetylases prevents cytokine-induced toxicity in beta cells. Diabetologia 50:779-789

15. Patel T, Patel V, Singh R, Jayaraman S (2011) Chromatin remodeling resets the immune system to protect against autoimmune diabetes in mice. Immunol Cell Biol 89:640-649

16. Asfari M, Janjic D, Meda P, Li G, Halban PA, Wollheim CB (1992) Establishment of 2-mercaptoethanol-dependent differentiated insulin-secreting cell lines. Endocrinology 130:167-178

17. Brunstedt J (1980) Rapid isolation of functionally intact pancreatic islets from mice and rats by percoll ${ }^{\mathrm{TM}}$ gradient centrifucation. Diabetes Metab 6:87-89

18. Nielsen R, Grontved L, Stunnenberg HG, Mandrup S (2006) Peroxisome proliferator-activated receptor subtype- and cell-typespecific activation of genomic target genes upon adenoviral transgene delivery. Mol Cell Biol 26:5698-5714

19. Willcox A, Richardson SJ, Bone AJ, Foulis AK, Morgan NG (2009) Analysis of islet inflammation in human type 1 diabetes. Clin Exp Immunol 155:173-181

20. Lagger G, O'Carroll D, Rembold M et al (2002) Essential function of histone deacetylase 1 in proliferation control and CDK inhibitor repression. EMBO J 21:2672-2681

21. Lei WW, Zhang KH, Pan XC et al (2010) Histone deacetylase 1 and 2 differentially regulate apoptosis by opposing effects on extracellular signal-regulated kinase 1/2. Cell Death Dis 1:e44

22. Lewis EC, Blaabjerg L, Storling J et al (2010) The oral histone deacetylase inhibitor ITF2357 reduces cytokines and protects islet beta-cells in vivo and in vitro. Mol Med 17:369-377

23. Christensen DP, Gysemans C, Dahllof M et al (2011) Inhibition of lysine deacetylase activity protects $\beta$-cells from inflammatory attack in vitro and in vivo by targeting NF-KB transcriptional activity. Diabetes 60:A132 (Abstract)

24. Chou DH, Bodycombe NE, Carrinski HA et al (2010) Smallmolecule suppressors of cytokine-induced beta-cell apoptosis. ACS Chem Biol 5:729-734

25. Storling J, Laman-Pillet N, Karlsen AE, Billestrup N, Bonny C, Mandrup-Poulsen T (2005) Antitumorigenic effect of proteasome inhibitors on insulinoma cells. Endocrinology 146:1718-1726

26. Mastrandrea LD, Sessanna SM, Laychock SG (2005) Sphingosine kinase activity and sphingosine-1 phosphate production in rat pancreatic islets and INS-1 cells: response to cytokines. Diabetes 54:1429-1436

27. Mosley AL, Ozcan S (2003) Glucose regulates insulin gene transcription by hyperacetylation of histone h4. J Biol Chem 278:19660-19666
28. Mosley AL, Ozcan S (2004) The pancreatic duodenal homeobox-1 protein $(\mathrm{Pdx}-1)$ interacts with histone deacetylases Hdac-1 and Hdac-2 on low levels of glucose. J Biol Chem 279:5424154247

29. Pautz A, Art J, Hahn S, Nowag S, Voss C, Kleinert H (2010) Regulation of the expression of inducible nitric oxide synthase. Nitric Oxide 23:75-93

30. Ashburner BP, Westerheide SD, Baldwin AS Jr (2001) The p65 (RelA) subunit of NF-kappaB interacts with the histone deacetylase (HDAC) corepressors HDAC1 and HDAC2 to negatively regulate gene expression. Mol Cell Biol 21:70657077

31. Kiernan R, Bres V, Ng RWM et al (2003) Post-activation TURN-OFf of NF-kappa B-dependent transcription is regulated by acetylation of p65. J Biol Chem 278:2758-2766

32. Dovey OM, Foster CT, Cowley SM (2010) Histone deacetylase 1 (HDAC1), but not HDAC2, controls embryonic stem cell differentiation. Proc Natl Acad Sci USA 107:8242-8247

33. Noh H, Oh EY, Seo JY et al (2009) Histone deacetylase-2 is a key regulator of diabetes- and transforming growth factor-beta1induced renal injury. Am J Physiol Renal Physiol 297:F729F739

34. Haumaitre C, Lenoir O, Scharfmann R (2008) Histone deacetylase inhibitors modify pancreatic cell fate determination and amplify endocrine progenitors. Mol Cell Biol 28:6373-6383

35. Lenoir O, Flosseau K, Ma FX et al (2011) Specific control of pancreatic endocrine $\beta$ - and $\delta$-cell mass by class IIa histone deacetylases HDAC4, HDAC5, and HDAC9. Diabetes 60:28612871

36. Christensen DP, Dahllof M, Lundh M et al (2011) Histone deacetylase (HDAC) inhibition as a novel treatment for diabetes mellitus. Mol Med 17:378-390

37. Dinarello CA, Fossati G, Mascagni P (2011) Histone deacetylase inhibitors for treating a spectrum of diseases not related to cancer. Mol Med 17:333-352

38. Leoni F, Zaliano A, Bertolini G et al (2001) The antitumor histone deacetylase inhibitor suberoylanilide hydroxamic acid exhibits anti-inflammatory properties via suppression of cytokines. Proc Natl Acad Sci 99:2995-3000

39. Lin HS, Hu CY, Chan HY et al (2007) Anti-rheumatic activities of histone deacetylase (HDAC) inhibitors in vivo in collagen-induced arthritis in rodents. Br J Pharmacol 150:862-872

40. Xiao C, Giacca A, Lewis GF (2011) Sodium phenylbutyrate, a drug with known capacity to reduce endoplasmic reticulum stress, partially alleviates lipid-induced insulin resistance and beta-cell dysfunction in humans. Diabetes 60:918-924

41. Ozcan U, Yilmaz E, Ozcan L et al (2006) Chemical chaperones reduce ER stress and restore glucose homeostasis in a mouse model of type 2 diabetes. Science 313:1137-1140

42. Eguchi K, Manabe I, Oishi-Tanaka Y et al (2012) Saturated fatty acid and TLR signaling link beta cell dysfunction and islet inflammation. Cell Metab 15:518-533 